\title{
Intracranial Aneurysms in Autosomal Dominant Polycystic Kidney Disease
}

\author{
M. Niemczyk, M. Gradzik, S. Niemczyk, M. Bujko, M. Gołębiowski, and L. Pączek
}

\begin{abstract}
BACKGROUND AND PURPOSE: ADPKD correlates with an increased frequency of ICANs, but universal screening for ICANs in patients with ADPKD is not currently recommended. The aim of our study was to determine which groups might benefit from screening by determining the prevalence of ICANs in the Polish ADPKD population and identifying any subgroups with an increased risk for ICANs.
\end{abstract}

MATERIALS AND METHODS: Eighty-three adult, predialysis-phase patients with ADPKD underwent screening for ICANs with MRA of the brain.

RESULTS: The prevalence of ICANs in the studied population was $16.9 \%$, with $6 \%$ of the screened group requiring neurosurgical intervention. We also found that the frequency of ICANs increases with age, reaching $22.4 \%$ in patients older than 45 years. All diagnosed ICANs were small $(<9 \mathrm{~mm})$ and were localized in the anterior circulation. In addition, MR imaging revealed arachnoid cysts in $4.8 \%$ of patients with ADPKD.

CONCLUSIONS: We suggest that patients older than 45 years with ADPKD be considered as candidates for screening for ICANs, and we propose a clinical algorithm for this subgroup. However, we could not find risk factors for ICANs in younger patients with ADPKD.

ABBREVIATIONS: ADPKD = autosomal dominant polycystic kidney disease; $\mathrm{AH}=$ arterial hypertension; $\mathrm{CKD}=$ chronic kidney disease; ICAN $=$ intracranial aneurysm; $\mathrm{Cl}$, confidence interval

A

DPKD is the most common monogenic disease, affecting 1 of every 1000 people. However, although ADPKD leads to endstage renal disease in a large proportion of patients, its symptoms are not limited to the kidney. Extrarenal manifestations include, among others, cysts in other organs (eg, liver and pancreas); arterial hypertension; and ICANs, ${ }^{1}$ which are more likely to occur in patients with ADPKD than in the general population. ${ }^{2}$ There is also a higher incidence of ICAN rupture in younger patients than is seen in the general population, ${ }^{3,4}$ which correlates with a high risk for serious complications or death. ${ }^{5,6}$

According to the analysis of Butler et al, ${ }^{7}$ universal screening for ICANs in the ADPKD population may positively influence patient outcome and is cost-effective. On the other hand, ICANs detected by screening are usually small, with a very low expected

Received September 6, 2012; accepted after revision November 13.

From the Departments of Immunology, Transplant Medicine, and Internal Diseases (M.N., L.P.) and Radiology (M. Gradzik, M. Gołebbiowski), Medical University of Warsaw, Warsaw, Poland; Department of Internal Diseases, Nephrology, and Dialysis (S.N.), Military Institute of Medicine, Warsaw, Poland; and Department of Neurosurgery (M.B.), The Medical Centre of Postgraduate Education, Warsaw, Poland.

Please address correspondence to Mariusz Niemczyk, Department of Immunology, Transplant Medicine and Internal Diseases, Medical University of Warsaw, Nowogrodzka 59, 02-006 Warsaw, Poland; e-mail: mariuszniemczyk@wp.pl

http://dx.doi.org/10.3174/ajnr.A3456 rupture rate. ${ }^{8}$ Therefore, in clinical practice, the indications for screening are limited to subgroups of patients with either a family history of ICAN or previous aneurysm rupture, those undergoing major elective surgery, or those in a high-risk occupation. ${ }^{9}$ Furthermore, universal screening is thought to be of low cost-effectiveness, and risks have been associated with treatment of diagnosed ICANs. ${ }^{10}$ Unfortunately, except for a positive family history of subarachnoid hemorrhage, no risk factors for ICANs in patients with ADPKD have been defined, ${ }^{3}$ which means that we are not able to identify all patients with a risk for subarachnoid hemorrhage from ICAN rupture. However, as some authors support systematic screening for ICANs in patients with $\mathrm{ADPKD},{ }^{11,12}$ our study was designed to determine the prevalence of ICANs in the Polish ADPKD population to identify subgroups with an increased risk for ICANs who might benefit from screening.

\section{MATERIALS AND METHODS}

Eighty-three patients diagnosed with ADPKD according to the unified ultrasonographic criteria ${ }^{13}$ were included in our study (Table 1). All included patients were white and were in the predialysis period of ADPKD. Twenty-three of the patients in this study were related to other patients, comprising 10 families. A detailed 
Table 1: Characteristics of study group

\begin{tabular}{ll}
\hline \multicolumn{1}{c}{ Characteristic } & \multicolumn{1}{c}{ Values } \\
\hline Men/Women (\%) & $32(38.6) / 51(61.4)$ \\
Mean age (range; SD) & $46(19-75 ; 15)$ \\
Stage of chronic kidney disease $(\%)^{\mathrm{a}}$ & \\
1 & $23(27.7)$ \\
2 & $20(24.1)$ \\
3 & $25(30.1)$ \\
4 & $14(16.9)$ \\
5 & $1(1.2)$ \\
Body mass index (\%) & \\
Underweight (BMI $\left.\leq 18.5 \mathrm{~kg} / \mathrm{m}^{2}\right)$ & $4(4.8)$ \\
Normal (BMI $\left.18.6-24.9 \mathrm{~kg} / \mathrm{m}^{2}\right)$ & $41(49.4)$ \\
Overweight (BMI $\left.25.0-29.9 \mathrm{~kg} / \mathrm{m}^{2}\right)$ & $28(33.7)$ \\
Obese (BMI $\left.\geq 30.0 \mathrm{~kg} / \mathrm{m}^{2}\right)$ & $10(12.1)$ \\
Arterial hypertension $(\%)$ & $80(96.4)$ \\
Insufficient control of blood pressure & $62(74.7)$ \\
\hline
\end{tabular}

Note:-BMI indicates body mass index.

a Estimated glomerular filtration rate is calculated according to the Chronic Kidney Disease Epidemiology Collaboration formula.

Table 2: Inclusion and exclusion criteria

\begin{tabular}{ll}
\hline \multicolumn{1}{c}{ Inclusion Criteria } & \multicolumn{1}{c}{ Exclusion Criteria } \\
\hline Diagnosis of ADPKD & Pregnancy \\
Age $\geq 18$ years & Contraindications for MR imaging \\
Written informed consent & $\begin{array}{c}\text { Dialysis or post-kidney } \\
\text { transplantation }\end{array}$ \\
\hline
\end{tabular}

list of the inclusion and exclusion criteria are presented in Table 2. A 3D time-of-flight MRA study of the brain was performed to screen for ICANs in patients included between May 2009 and June 2012 at the Medical University of Warsaw Department of Radiology. The MR scanners used in the study were a Magnetom Avanto 1.5T (Siemens, Erlangen, Germany) and an Ingenia 1.5T HP (Philips Healthcare, Best, the Netherlands). For the Magnetom Avanto, examinations were made between 2009 and 2011; for the Ingenia, examinations were conducted in 2012. Details of the imaging parameters are presented in Table 3. For patients with a suspected ICAN, MR results were verified by use of CT angiography and were then referred to a specialist in neurosurgery. The criteria used for intervention in patients with intact aneurysms were an aneurysmal diameter of more than $5 \mathrm{~mm}$, irregular shape with blebs or domes on the aneurysm, or progression in size or change in shape on CT angiography. In addition, established high-risk factors for aneurysmal rupture-including a history of tobacco use, hypertension, and a family history of intracranial aneurysm ruptures-were considered in the assessment of treatment options.

This study was conducted in accordance to the principles of the Declaration of Helsinki, and the ethics committee of the Med- ical University of Warsaw approved the protocol. All patients gave written informed consent for inclusion into the study.

We performed statistical analysis by using Statistica software, version 10 (StatSoft, Tulsa, Oklahoma). $\chi^{2}$ tests and Fisher exact tests were used when appropriate. A $P$ value of $<.05$ was considered statistically significant.

\section{RESULTS}

On the basis of MRA, 18 ICANs in 14 patients were suspected. Of those, CT angiography confirmed 16 ICANs in 12 patients. Aneurysms were not proven in 2 patients with an MR imaging suspicion of very small ( $\leq 2 \mathrm{~mm}$ ) ICAN. In 2 male patients with previous ICAN rupture (both occurring before age 40 years), new aneurysms were not diagnosed. As a result, we determined that the prevalence of ICANs in the studied population was $16.9 \%$ (14/83; 95\% CI, 8.6\%-25.1\%). Furthermore, in 4 patients $(28.6 \%) 2$ ICANs were diagnosed, with the remainder (10/14; $71.4 \%$ ) found to have a single ICAN. All ICANs were localized in the anterior circulation, including $12(66.7 \%)$ in the middle cerebral artery, $5(27.8 \%)$ in the anterior communicating artery, and 1 $(5.6 \%)$ in the internal carotid artery. Among 16 newly diagnosed ICANs, 9 were smaller than $5 \mathrm{~mm}$, and the remaining 7 were between 5 and $9 \mathrm{~mm}$ in diameter. After neurosurgical consultation, 5 patients with ICANs $\geq 5 \mathrm{~mm}$ in diameter were treated, and the remaining 7 were not. In total, $6 \%$ (95\% CI, 0.8\%-11.3\%) of screened patients with ADPKD required treatment of ICANs. In addition, there was no significant difference in the prevalence of ICANs across sex (women: 9/51 [17.6\%]; 95\% CI, 6.8\%-28.5\%; men: $5 / 32$ [15.6\%]; 95\% CI, 2.3\%-28.9\%) in patients with ADPKD.

The rates of newly diagnosed ICANs were recorded in the second $(0 / 1 ; 0 \%)$, third $(0 / 15 ; 0 \%)$, fourth $(1 / 14 ; 7.1 \%)$, fifth $(1 / 11$; $7.1 \%)$, sixth $(7 / 27 ; 25.9 \%)$, seventh $(3 / 9 ; 33.3 \%)$, and eighth $(0 / 6$; $0 \%)$ decades of life $(P<.05$ for differences between the third and sixth and the third and seventh decades of life), that is, newly diagnosed ICANs were detected in $1(2.9 \%)$ of 34 patients $\leq 45$ years old and in $11(22.4 \%)$ of 49 patients $>45$ years old $(P<.05)$. In addition, the only patient younger than 45 years diagnosed with ICAN did not need treatment, whereas in patients older than 45 years in which ICANs were detected, 5 (45.4\%) of 11 did. New ICANs were diagnosed in $0(0 \%)$ of 4 patients, $5(12.2 \%)$ of 41 patients, $4(14.3 \%)$ of 28 patients, and $3(30 \%)$ of 10 patients who were underweight, normal weight, overweight, and obese, respectively. However, these differences did not reach statistical significance. Furthermore, new ICANs were diagnosed in patients with first $(3 / 23 ; 13 \%)$, second $(1 / 20 ; 5 \%)$, third $(3 / 25 ; 12 \%)$, fourth

Table 3: Imaging parameters used for 3D TOF MRA

\begin{tabular}{|c|c|c|}
\hline & \multicolumn{2}{|c|}{ MR Imaging Scanner } \\
\hline & Siemens Magnetom Avanto 1.5T & Philips Ingenia 1.5T HP \\
\hline Years of use & 2009-2011 & 2012 \\
\hline Sequence type & TIWI 3D GRE & TIWI 3D FFE \\
\hline TOF sequence parameters & TE: 7.15 ms; TR: 22 ms; NSA: 1 & TE: 6.91 (outphase) ms; TR: 25 ms; NSA: 1 \\
\hline Pixel size (mm) MPS & $0.7 / 0.7 / 0.7$ & $0.4 / 0.7 / 1.0$ \\
\hline Matrix RECON & 320 & 512 \\
\hline Flip angle $\left({ }^{\circ}\right)$ & 25 & 20 \\
\hline Total scan duration (min/sec) & $7 / 13$ & $4 / 50$ \\
\hline
\end{tabular}

FFE indicates fast-field echo; GRE, gradient-recalled echo; MPS, measurement phase and section direction; NSA, number of sampling averages. 
(5/14; 35.7\%), and fifth $(0 / 1 ; 0 \%)$ stage CKD $(P<.05$ for the difference between second and fourth stage CKD).

New ICANs were detected in $7(11.3 \%)$ of 62 patients with insufficiently controlled AH, 4 (22.2\%) of 18 patients with wellcontrolled $\mathrm{AH}$, and 1 (33.3\%) of 3 patients without $\mathrm{AH}$, though these differences were not statistically significant. Among the 23 patients with family members in the studied population, ICANs were detected in 2 patients from different families. In addition, MR imaging results revealed arachnoid cysts in 4 patients ( 2 men, 2 women; $4.8 \%$; 95\% CI, 0.1-9.5\%), though no patient with an arachnoid cyst was found to have an ICAN. From an anatomic standpoint, 3 cysts were localized in the posterior cranial fossa and 1 in the middle cranial fossa; the latter was the largest cyst $(50 \times$ $48 \times 90 \mathrm{~mm}$ ). None were determined to be symptomatic or require treatment.

\section{DISCUSSION}

In our study, the prevalence of ICANs in the ADPKD population was $16.9 \%$ (95\% CI, 8.6\%-25.1\%), with equal distribution between male and female patients, which is consistent with what is found in the literature $\left(4 \%{ }^{14}-22.5 \%{ }^{15}\right)$. However, our findings are slightly higher compared with the large study of Xu et al, ${ }^{11}$ in which the prevalence of ICANs in ADPKD was $12.4 \%$, with equal distribution between the sexes. This feature may be able to distinguish between ICANs in patients with ADPKD and ICANs in the general population, where a higher prevalence has been reported in women. ${ }^{2}$ Of note, only $6 \%$ (95\% CI, $\left.0.8-11.3 \%\right)$ of screened patients with ADPKD required treatment of ICANs. However, when the prevalence of ADPKD is considered, the number of patients who need treatment becomes substantial, accounting for 60 patients for each 1 million people in the general population.

All ICANs detected in our study were small, which is in accordance with the study of $\mathrm{Xu}$ et al. ${ }^{11}$ However, because $50 \%$ of ICANs that rupture are $<10 \mathrm{~mm},{ }^{4,5}$ small diameter is not a consideration against screening. All ICANs detected in our study were localized in the anterior circulation, in accordance with a previous study. ${ }^{11}$ The most common site of ICAN in ADPKD is the middle cerebral artery, another feature that distinguishes ICANs in patients with ADPKD from ICANs in the general population, where the internal carotid artery is the most common location. ${ }^{3} \mathrm{Al}-$ though no case of ICAN in the posterior circulation was detected in our study, some authors have reported this location as being $\operatorname{rare}^{4,5,8,15}$ and therefore it should be expected in some cases. In addition, we found multiple ICANs in $28.6 \%$ of patients, which is more than what has been previously reported., ${ }^{4,5,11}$

According to our data, the prevalence of ICANs increases with age. We only found a single ICAN in patients younger than 45 years, whereas in patients older than 45 years, the frequency of ICANs increased to $22.4 \%$. Because almost half of them required treatment, in our opinion, screening for ICANs should be considered in all patients older than 45 years with ADPKD. An additional reason is that a large proportion of such patients in this age group go on to have end-stage renal disease ${ }^{1}$ and become candidates for kidney transplantation or other surgical procedures, such as nephrectomy. Because ICAN is often seen together with uncontrolled blood pressure (occurring in nearly three-quarters of patients in our group), which may increase the risk for periop- erative complications, we suggest screening for ICANs with MRA as an obligatory procedure during pretransplant evaluation in patients with ADPKD. Other authors have also reported increases in ICAN frequency with age and have suggested systematic screening in patients with ADPKD, but they recommend that screenings start at age 30 years. ${ }^{11}$ However, we believe that screening at this young age is not supported by our results. We also did not find a connection between the prevalence of ICANs and body mass index. In addition, although we tried to assess the connection between the prevalence of ICANs and renal function, we only found statistically significant differences between second and fourth stage CKD. This finding is similar to a previous study, ${ }^{11}$ which found the frequency of ICANs to be unrelated to renal function.

$\mathrm{AH}$ is a risk factor for subarachnoid hemorrhage in the general population, ${ }^{16}$ but hypertension is not considered a risk factor for the formation of ICANs in ADPKD. ${ }^{3,11}$ In our study, we found that insufficient control of AH did not correlate with an increased frequency of ICANs. However, the limitations of our analysis were that the number of "well-controlled AH" and "no-AH" subgroups was insufficient and that only qualitative data on hypertension were considered. The prevalence of ICANs has been previously reported to be associated with the duration of hypertension. ${ }^{11}$ However, in our experience it is a difficult factor to assess, as many patients with ADPKD also live with unrecognized AH. Additional risk factors for subarachnoid hemorrhage in the general population are tobacco smoking and heavy alcohol consumption, ${ }^{16}$ but we omitted these factors in our analysis because of incomplete data.

According to published data, subarachnoid hemorrhage from rupture of ICAN in ADPKD often occurs at a young age, ${ }^{5,15,17}$ with $64 \%$ occurring before age 50 years. ${ }^{4}$ Also, patients with a previous stroke from a ruptured ICAN are at risk for new ICANs and should be examined for them. ${ }^{18}$ We only detected 2 patients with previous rupture of ICAN in our study, and we did not detect new ICANs in these patients. Although we believe that the number of patients in our subgroup was too low to make valuable conclusions, we agree that this group should be periodically screened for new ICANs. Still the question remains of how to predict an ICAN rupture in younger patients; unfortunately, we failed to identify any risk factors for ICAN and rupture in younger patients with ADPKD.

A positive family history for ICAN is considered a risk factor for ICAN, ${ }^{5,11,19}$ and ICAN rupture tends to cluster in some families. ${ }^{11,19}$ In our study, we did not find an increased prevalence in family members of involved patients. Again, we believe that this finding is probably the result of a low number of patients in this particular subgroup.

MRA revealed false-positive results in 2 of our patients. Although MRA is a method of choice in the detection of ICANs in patients with ADPKD because there is no x-ray exposure or need for contrast media administration, it has limited specificity, especially in the detection of very small ICANs. Although such ICANs usually do not need treatment, false-positive results may lead to redundant CTA. Therefore, the suspicion of very small ICANs should not be verified with CTA but may only require periodic follow-ups with MRA and should only be verified in case of en- 
largement. Additional findings included the discovery of arachnoid cysts in $4.8 \%$ of patients. Previous reports have found that arachnoid cysts in ADPKD occur at a rate of $7.1 \%-8.1 \% .^{20,21}$ Although these cysts increase the risk for chronic subdural hematoma, ${ }^{22}$ none of the discovered cysts resulted in neurologic symptoms or complications.

We propose a clinical algorithm for ICANs in ADPKD, in which all patients older than 45 years with ADPKD undergo screening with MRA, after which the suspicion of an ICAN $>2$ $\mathrm{mm}$ in diameter should be followed by CTA, and if verified positively, the patient should be referred to a specialist in neurosurgery. In patients with suspected ICAN $\leq 2 \mathrm{~mm}$ in diameter, follow-up with MRA should be considered. Although we do not propose any specific intervals between MR imaging, we believe that that once a year seems reasonable. The obvious limitation of our algorithm is that at-risk patients younger than 45 years are not included, so indications for screening in that group still need to be established.

\section{CONCLUSIONS}

The prevalence of ICANs in a sample of Polish patients with ADPKD amounts to $16.9 \%$. Furthermore, $6 \%$ of the screened population required neurosurgical treatment of ICANs. We also found that the frequency of ICANs increases with age, reaching $22.4 \%$ in patients older than 45 years. Therefore, we suggest that this subgroup of patients be considered as candidates for ICAN screening, especially before elective surgery such as nephrectomy or kidney transplantation. Unfortunately, we could not determine any significant risk factors for ICANs in patients younger than 45 years with ADPKD.

\section{REFERENCES}

1. Chang MY, Ong AC. Autosomal dominant polycystic kidney disease: recent advances in pathogenesis and treatment. Nephron Physiol 2008;108:1-7

2. Vlak MH, Algra A, Brandenburg R, et al. Prevalence of unruptured intracranial aneurysms, with emphasis on sex, age, comorbidity, country, and the time period: a systematic review and meta-analysis. Lancet Neurol 2011;10:626-36

3. Rinkel GJ. Intracranial aneurysm screening: indications and advice for practice. Lancet Neurol 2005;4:122-28

4. Schievink WI, Torres VE, Piepgras DG, et al. Saccular intracranial aneurysms in autosomal dominant polycystic kidney disease. J Am Soc Nephrol 1992;3:88-95

5. Gieteling EW, Rinkel GJ. Characteristics of intracranial aneurysms and subarachnoid heamorrhage in patients with polycystic kidney disease. J Neurol 2003;250:418-23

6. Zuka M, Onoe T, Kawano M, et al. Sudden death of a young male with previously undiagnosed autosomal dominant polycystic kidney disease (ADPKD). Leg Med (Tokyo) 2011;13:35-38

7. Butler WE, Barker FG 2nd, Crowell RM. Patients with polycystic kidney disease would benefit from routine magnetic resonance angiographic screening for intracerebral aneurysms: a decision analysis. Neurosurgery 1996;38:506-15

8. Irazabal MV, Huston J 3rd, Kubly V, et al. Extended follow-up of unruptured intracranial aneurysms detected by presymptomatic screening in patients with autosomal dominant polycystic kidney disease. Clin J Am Soc Nephrol 2011;6:1274-85

9. Torres VE, Pirson Y, Wiebers DO. Cerebral aneurysms. N Engl J Med 2006;355:2703-04; author reply 2705

10. Hughes PD, Becker GJ. Screening for intracranial aneurysms in autosomal dominant polycystic kidney disease. Nephrology (Carlton) 2003;8:163-70

11. Xu HW, Yu SQ, Mei CL, et al. Screening for intracranial aneurysm in 355 patients with autosomal-dominant polycystic kidney disease. Stroke 2011;42:204-06

12. Mariani L, Bianchetti MG, Schroth G, et al. Cerebral aneurysms in patients with autosomal dominant polycystic kidney disease-to screen, to clip, to coil? Nephrol Dial Transplant 1999;14:2319-22

13. Pei Y, Obaji J, Dupuis A, et al. Unified criteria for ultrasonographic diagnosis of ADPKD. J Am Soc Nephrol 2009;20:205-12

14. Chapman $A B$, Rubinstein $D$, Hughes R, et al. Intracranial aneurysms in autosomal dominant polycystic kidney disease. $N$ Engl J Med 1992;327:916-20

15. Belz MM, Fick-Brosnahan GM, Hughes RL, et al. Recurrence of intracranial aneurysms in autosomal-dominant polycystic kidney disease. Kidney Int 2003;63:1824-30

16. Juvela S. Prevalence of and risk factors for intracranial aneurysms. Lancet Neurol 2011;10:595-97

17. Kubo S, Nakajima M, Fukuda K, et al. A 4-year-old girl with autosomal dominant polycystic kidney disease complicated by a ruptured intracranial aneurysm. Eur J Pediatr 2004;163:675-77

18. Chauveau D, Sirieix ME, Schillinger F, et al. Reccurent rupture of intracranial aneurysms in autosomal dominant polycystic kidney disease. BMJ 1990;301:966-67

19. Huston J 3rd, Torres VE, Sulivan PP, et al. Value of magnetic resonance angiography for the detection of intracranial aneurysms in autosomal dominant polycystic kidney disease. J Am Soc Nephrol 1993;3:1871-77

20. Romão EA, Moysés Neto M, Teixeira SR, et al. Renal and extrarenal manifestations of autosomal dominant polycystic kidney disease. Braz J Med Biol Res 2006;39:533-38

21. Schievink WI, Huston J 3rd, Torres VE, et al. Intracranial cysts in autosomal dominant polycystic kidney disease. I Neurosurg 1995;83:1004-07

22. Wijdicks EF, Torres VE, Schievink WI. Chronic subdural hematoma in autosomal dominant polycystic kidney disease. Am J Kidney Dis 2000;35:40-43 